\title{
WEIGHTED DECAY ESTIMATE FOR THE WAVE EQUATION
}

\author{
VALERY COVACHEV AND VLADIMIR GEORGIEV
}

(Communicated by Barbara L. Keyfitz)

\begin{abstract}
The work is devoted to the proof of a new $L^{\infty}-L^{2}$ weighted estimate for the solution to the nonhomogeneous wave equation in $(3+1)$-dimensional space-time. The weighted Sobolev spaces are associated with the generators of the Poincare group. The estimate obtained is applied to prove the global existence of a solution to a nonlinear system of wave and Klein-Gordon equations with small initial data.
\end{abstract}

\section{INTRODUCTION}

Essential results concerning the global existence of solution to the $(3+1)$ dimensional nonlinear wave equation have been obtained recently by Klainerman $[9,10]$, Hörmander [6, 7] (see also [1-4]). The use of Sobolev weighted spaces associated with the generators

$$
\begin{array}{cc}
\partial_{t}, \partial_{j}=\partial / \partial x_{j}, & j=1,2,3, \\
\Omega_{j k}=x_{j} \partial_{k}-x_{k} \partial_{j}, & 1 \leq j<k \leq 3, \\
\Omega_{0 j}=t \partial_{j}+x_{j} \partial_{t}, & j=1,2,3,
\end{array}
$$

of the Poincare group in $\mathbf{R}^{4}$ plays the key role in the approach developed by Klainerman and Hörmander. Denote the above generators by $\Gamma_{1}, \ldots, \Gamma_{10}$.

One of the sharpest estimates of the $L^{\infty}$-norm of the solution to the nonhomogeneous wave equation is obtained by Hörmander in [7]. This estimate improves some previous estimates due to Klainerman $[9,10]$. More precisely, if $u(t, x)$ is a solution to the Cauchy problem

$$
\square u=f, \quad \square=\partial_{t}^{2}-\sum_{j=1}^{3} \partial_{j}^{2}, \quad u(0, x)=\partial_{t} u(0, x)=0
$$

and $f(t, x) \in C^{\infty}\left(\mathbf{R}_{+} ; \mathscr{S}\left(\mathbf{R}^{3}\right)\right)$, then the estimate derived in the Appendix of

Received by the editors June 5, 1989 and, in revised form, June 1, 1990.

1980 Mathematics Subject Classification (1985 Revision). Primary 35L05, 35L70; Secondary $35 \mathrm{G} 25$.

Key words and phrases. Decay estimate, wave equation. 
[7] implies

$$
(1+t+|x|)|u(t, x)| \leq C \sum_{|\beta|+j \leq 2} \int_{0}^{t} \int_{\mathbf{R}^{3}}\left|\Gamma^{\beta} L_{0}^{j} f(s, y)\right| \frac{d y d s}{(1+s+|y|)} .
$$

Here $L_{0}=t \partial_{t}+\sum_{j=1}^{3} x_{j} \partial_{j}$ is the radial vector field.

On the other hand, some important coupled systems of nonlinear wave and Klein-Gordon equations have been investigated in [1-4]; these show that the use of the radial vector field $L_{0}$ is not convenient for the proof of a global existence theorem. To overcome this difficulty, in $[2,3]$ another $L^{\infty}-L^{2}$ weighted estimate is proposed, when the inclusion

$$
\operatorname{supp} f(s, y) \subseteq\{|y| \leq s+R\}
$$

is fulfilled for some constant $R>0$. The estimate has the form

$$
(1+t)|u(t, x)| \leq C \sum_{r=0}^{\infty} \sum_{|\beta| \leq 3} \sup _{s \in I_{r} \cap[0, t]}(1+s)^{3 / 2}\left\|\Gamma^{\beta} f(s)\right\| .
$$

Here $I_{r}$ is the interval $\left[2^{r-1}, 2^{r+1}\right]$ for $r \geq 1, I_{0}=[0,2]$ and $\|g(s)\|$ denotes the norm

$$
\|g(s)\|=\left(\int_{\mathbf{R}^{3}}|g(s, y)|^{2} d y\right)^{1 / 2} .
$$

The main goal of this work is to obtain an improved estimate of type (1.5) for the solution $u(t, x)$ to the Cauchy problem (1.2), provided assumption (1.4) is not fulfilled.

Such an estimate will be crucial for the proof of the global existence of solution to some important nonlinear systems of wave and Klein-Gordon equations, when the initial data are not compactly supported (see the approach developed in $[1-4])$.

\section{MAIN RESULT}

Given any function $f(s, y) \in C^{\infty}\left(\mathbf{R}_{+}, \mathscr{S}\left(\mathbf{R}^{3}\right)\right)$ consider the weighted $L^{2}$ norm

$$
\|f(s)\|_{k}=\left(\int_{\mathbf{R}^{3}}(1+s+|y|)^{2 k}|f(s, y)|^{2} d y\right)^{1 / 2} ;
$$

and, analogously, for $f(y) \in \mathscr{S}\left(\mathbf{R}^{3}\right)$, denote

$$
\|f\|_{k}=\left(\int_{\mathbf{R}^{3}}(1+|y|)^{2 k}|f(y)|^{2} d y\right)^{1 / 2}
$$

If $k=0$, then we omit the index $k$ and write simply $\|f(s)\|$ and $\|f\|$.

Theorem 1. Let $f(s, y) \in C^{\infty}\left(\mathbf{R}_{+} ; \mathscr{S}\left(\mathbf{R}^{3}\right)\right)$ and let $u(t, x)$ be a solution to the Cauchy problem (1.2). Then we have

$$
(1+t+|x|)|u(t, x)| \leq C \sum_{r=0}^{\infty} \sum_{|\beta| \leq 3} \sup _{s \in I_{r} \cap[0, t]}\left\|\Gamma^{\beta} f(s)\right\|_{3 / 2} .
$$


Taking advantage of the fact that the generators $\Gamma_{j}$ commute with the operator $\square$, we obtain the following.

Corollary 2. Under the assumptions of Theorem 1 we have

$$
(1+t+|x|)\left|\Gamma^{\alpha} u(t, x)\right| \leq C \sum_{r=0}^{\infty} \sum_{|\beta| \leq 3+|\alpha|} \sup _{s \in I_{r} \cap[0, t]}\left\|\Gamma^{\beta} f(s)\right\|_{3 / 2} .
$$

Combining the above estimates with the estimates due to W. von Wahl [11], one can treat also the case when the initial data of the solution to (1.2) are nonzero. Namely, assuming $u(0, x)=\varepsilon f(x)$ and $\partial_{t} u(0, x)=\varepsilon g(x), f, g \in$ $\mathscr{S}\left(\mathbf{R}^{3}\right)$, for any positive real number $\delta>0$ we have

$$
(1+t+|x|)\left|\Gamma^{\alpha} u(t, x)\right| \leq C \varepsilon+C \sum_{|\beta| \leq 3+|\alpha|} \sup _{s \in[0, t]}\left\|\Gamma^{\beta} f(s)\right\|_{3 / 2+\delta} .
$$

\section{Preliminaries}

The main goal of this section is to obtain some estimates of the Radon transform

$$
\mathscr{R}(f)(\rho, \omega)=\int_{x \omega=\rho} f(x) d S_{x}, \quad \rho \in \mathbf{R}, \omega \in \mathbf{S}^{2}
$$

of a function $f(x) \in \mathscr{S}\left(\mathbf{R}^{3}\right)$.

Lemma 3.1. Suppose $f(x) \in C_{0}^{\infty}\left(\mathbf{R}^{3}\right)$ and $\operatorname{supp} f \subseteq\{|x| \leq R\}$. Then we have the estimate

$$
\sup _{\rho \in \mathbf{R}, \omega \in \mathbf{S}^{2}}|\mathscr{R}(f)(\rho, \omega)| \leq C(1+R)^{1 / 2} \sum_{|\beta| \leq 2}\left\|\Omega^{\beta} f\right\|,
$$

where $\Omega^{\beta}=\Omega_{1}^{\beta_{1}} \Omega_{2}^{\beta_{2}} \Omega_{3}^{\beta_{3}}$ and $\Omega_{1}, \Omega_{2}, \Omega_{3}$ are the generators $\Omega_{j k}=x_{j} \partial_{k}-x_{k} \partial_{j}$, $1 \leq j<k \leq 3$, of the group of rotations on $\mathbf{R}^{3}$.

Proof. The assumption supp $f \subseteq\{|x| \leq R\}$ implies that

$$
\operatorname{supp}_{\rho} \mathscr{R}(f)(\rho, \omega) \subseteq\{|\rho| \leq R\}
$$

and we get the inequality

$$
|\mathscr{R}(f)(\rho, \omega)| \leq C \int_{-R}^{R}\left|\partial_{\rho} \mathscr{R}(f)(\rho, \omega)\right| d \rho .
$$

Applying the Cauchy-Schwartz inequality we get

$$
|\mathscr{R}(f)(\rho, \omega)| \leq C(1+R)^{1 / 2}\left(\int_{-R}^{R}\left|\partial_{\rho} \mathscr{R}(f)(\rho, \omega)\right|^{2} d \rho\right)^{1 / 2} .
$$

The Sobolev inequality on $\mathbf{S}^{2}$ gives

$$
|g(\omega)|^{2} \leq C \sum_{|\beta| \leq 2} \int_{\mathbf{S}^{2}}\left|\Omega^{\beta} g(\theta)\right|^{2} d \theta .
$$


Combining the above estimates with the Plancherel identity (see [5])

$$
\int_{-\infty}^{\infty} \int_{\mathbf{S}^{2}}\left|\partial_{\rho} \mathscr{R}(f)(\rho, \omega)\right|^{2} d \omega d \rho=c\|f\|^{2},
$$

we get the needed estimate.

This completes the proof.

Corollary 3.2. Suppose $f(x) \in C_{0}^{\infty}\left(\mathbf{R}^{3}\right)$ and

$$
\operatorname{supp} f(x) \subseteq\left\{c_{1} R \leq|y| \leq c_{2} R\right\}
$$

for some positive constants $c_{1}, c_{2}$ and $R \geq 1$. Then we have

$$
|\mathscr{R}(f)(\rho, \omega)| \leq C \sum_{|\beta| \leq 2}\left\|\Omega^{\beta} f\right\|_{1 / 2} .
$$

The next step in this section is the analysis of the case when the function $f(x)$ is not compactly supported.

Lemma 3.3. Let $f(x) \in \mathscr{S}\left(\mathbf{R}^{3}\right)$. Given any $R>0$, we have the estimate

$$
|\mathscr{R}(f)(\rho, \omega)| \leq C(1+R)^{-1 / 2} \sum_{|\beta| \leq 2}\left\|\Omega^{\beta} f\right\|_{1}+C(1+R)^{1 / 2} \sum_{|\beta| \leq 2}\left\|\Omega^{\beta} f\right\| .
$$

Proof. First, consider the case $|\rho| \geq 1+R$. Then the relation

$$
\mathscr{R}(f)(\rho, \omega)=\rho^{-1} \int_{0}^{\rho} \partial_{\tau}(\tau \mathscr{R}(f)(\tau, \omega)) d \tau
$$

and the Cauchy-Schwartz inequality yield

$$
|\mathscr{R}(f)(\rho, \omega)| \leq \rho^{-1 / 2}\left(\int_{0}^{\rho}\left|\partial_{\tau}(\tau \mathscr{R}(f)(\tau, \omega))\right|^{2} d \tau\right)^{1 / 2} .
$$

Applying the Sobolev inequality on $\mathbf{S}^{2}$, we find

$$
|\mathscr{R}(f)(\rho, \omega)| \leq C(1+R)^{-1 / 2} \sum_{|\beta| \leq 2}\left(\int_{\mathbf{S}^{2}} \int_{0}^{\rho}\left|\partial_{\tau}\left(\tau \mathscr{R}\left(\Omega^{\beta} f\right)(\tau, \theta)\right)\right|^{2} d \tau d \theta\right)^{1 / 2} .
$$

Since

$$
\tau \mathscr{R}(g)(\tau, \theta)=\tau \int_{y \cdot \theta=\tau} g(y) d S_{y}=\sum_{j=1}^{3} \theta_{j} \mathscr{R}\left(y_{j} g\right)(\tau, \theta),
$$

we conclude that

$$
\left|\tau \partial_{\tau}(\mathscr{R}(g)(\tau, \theta))\right| \leq C \sum_{j=1}^{3}\left|\partial_{\tau} \mathscr{R}\left(y_{j} g\right)(\tau, \theta)\right|,
$$

and via the Plancherel identity (3.2) we get

$$
|\mathscr{R}(f)(\rho, \omega)| \leq C(1+R)^{-1 / 2} \sum_{|\beta| \leq 2}\left\|\Omega^{\beta} f\right\|_{1} .
$$


The identity (3.1) shows that $\mathscr{R}(f)(\rho, \omega)$ is an odd function. Therefore, to finish the proof it is sufficient to consider only the case $0 \leq \rho \leq R+1$. If this is the case, then we apply the estimate

$$
|\mathscr{R}(f)(\rho, \omega)-\mathscr{R}(f)(1+R, \omega)| \leq \int_{0}^{1+R}\left|\partial_{\tau} \mathscr{R}(f)(\tau, \omega)\right| d \tau .
$$

Applying the arguments given in the proof of Lemma 3.1, we obtain

$$
\int_{0}^{R}\left|\partial_{\tau} \mathscr{R}(f)(\tau, \omega)\right| d \tau \leq C(1+R)^{1 / 2} \sum_{|\beta| \leq 2}\left\|\Omega^{\beta} f\right\|
$$

This estimate and (3.4) complete the proof.

\section{Proof of Theorem 1}

We lose no generality assuming that $f$ is a real-valued function.

First, consider the case $|x| \geq t / 2$. Then we can use the following representation of the solution to the wave equation obtained in [2] (see Lemma 3.1 in [2]),

$$
\begin{aligned}
|x| u(t, x)= & -2 \pi \sum_{\sigma= \pm 1} \sigma \operatorname{Re} \int_{0}^{\infty} \hat{f}(\rho, \sigma \rho \omega, t) e^{i \rho(t+\sigma|x|)} d \rho \\
& +\sum_{1 \leq r<k \leq 3} \operatorname{Re} \int_{\mathbf{R}^{3}} \frac{c_{k r}(x, \xi)}{|\omega \times \xi|} e^{i(t|\xi|+x \cdot \xi)} \hat{f}_{k r}(|\xi|, \xi, t) \frac{d \xi}{|\xi|},
\end{aligned}
$$

where $\omega=x /|x|, \hat{f}_{k r}(\tau, \xi, t)=\xi_{k} \partial_{\xi_{r}} \hat{f}-\xi_{r} \partial_{\xi_{k}} \hat{f}, \hat{f}(\tau, \xi, t)$ is the Fourier transform of the function $f(s, y) \chi(0 \leq s \leq t)$, i.e.

$$
\hat{f}(\tau, \xi, t)=\int_{0}^{t} \int_{\mathbf{R}^{3}} f(s, y) e^{-i(\tau s+\xi \cdot y)} d y d s .
$$

Moreover, $c_{k r}(x, \xi)$ are bounded functions, homogeneous of degree 0 with respect to $x, \xi$ and $a \times b$ denotes the vector product in $\mathbf{R}^{3}$.

If $|x| \leq t / 2$, then we use a similar representation derived in [2] (see Lemma 3.3 in $[2])$

$$
\begin{array}{r}
u(t, x)=\operatorname{Re} \sum_{|\beta| \leq 1} \int_{0}^{\infty} \int_{S^{2}}(t+x \cdot \omega)^{-1} c_{\beta}(\omega)\left(\Gamma^{\beta} f\right)^{\wedge}(\rho, \rho \omega, t) e^{i \rho(t+x \cdot \omega)} d \omega d \rho \\
-\operatorname{Re} \sum_{j=1}^{3} \int_{0}^{\infty} \int_{S^{2}}(t+x \cdot \omega)^{-1} \omega_{j}\left(\tilde{f}_{j}(t, \rho \omega)-e^{i \rho t} \tilde{f}_{j}(0, \rho \omega)\right) e^{i \rho x \cdot \omega} d \omega d \rho .
\end{array}
$$

Here $(G)^{\wedge}(\tau, \xi, t)$ denotes the Fourier transform of the function $G(t, y)$ determined by (4.2), $\tilde{f}_{j}$ is the partial Fourier transform of the function $y_{j} f(t, y)$ with respect to the spatial variables, i.e.

$$
\tilde{f}_{j}(t, \xi)=\int_{\mathbf{R}^{3}} y_{j} f(t, y) e^{-i y \cdot \xi} d y .
$$


In the sequel, we shall use also the following simple relation between the Fourier transform of a real-valued function and its Radon transform (see [5], [2])

$$
2 \operatorname{Re} \int_{0}^{\infty} \widetilde{F}(t, \rho \omega) e^{i \rho \tau} d \rho=\mathscr{R}(F)(\tau, \omega, t),
$$

where

$$
\mathscr{R}(F)(\tau, \omega, t)=\int_{y . \omega=\tau} F(t, y) d S_{y}
$$

is the Radon transform with respect to the spatial variables. The above relation yields

$$
2 \operatorname{Re} \int_{0}^{\infty} \widehat{F}(\rho, \rho \omega, t) e^{i \rho \tau} d \rho=\int_{0}^{t} \mathscr{R}(F)(\tau-s, \omega, s) d s
$$

for any real-valued function $F(s, y) \in C^{\infty}\left(\mathbf{R}_{+} ; \mathscr{S}\left(\mathbf{R}^{3}\right)\right)$.

In the case $|x| \geq t / 2$ we use the identities (4.1), (4.4) and derive the estimate

$$
(1+t+|x|)|u(t, x)| \leq C \sup _{(\tau, \omega) \in \mathbf{R} \times \mathbf{S}^{2}} \sum_{|\beta| \leq 1} \int_{0}^{t}\left|\mathscr{R}\left(\Gamma^{\beta} f\right)(\tau-s, \omega, s)\right| d s .
$$

The application of Lemma 3.3 with $R=s$ gives

$$
\begin{aligned}
(1+t+|x|)|u(t, x)| \leq & C \int_{0}^{t} \sum_{|\beta| \leq 3}\left\|\Gamma^{\beta} f(s)\right\|_{1 / 2} d s \\
& +C \int_{0}^{t}(1+s)^{-1 / 2} \sum_{|\beta| \leq 3}\left\|\Gamma^{\beta} f(s)\right\|_{1} d s .
\end{aligned}
$$

Taking advantage of the simple estimate

$$
\int_{0}^{t} \phi(s) d s \leq C \sum_{r=0}^{\infty} \sup _{s \in I_{r} \cap[0, t]}(1+s)|\phi(s)|,
$$

we obtain the desired estimate.

Finally, let us turn to the case $|x| \leq t / 2$. Now we use the relations (4.3), (4.4), (4.5), together with Lemma 3.3, and derive

$$
\begin{aligned}
(1+t)|u(t, x)| \leq & C \int_{0}^{t}(1+s)^{-1 / 2} \sum_{|\beta| \leq 3}\left\|\Gamma^{\beta} f(s)\right\|_{1} d s \\
& +C \sum_{j=1}^{3} \sup _{(\tau, \omega) \in \mathbf{R} \times \mathbf{S}^{2}}\left|\mathscr{R}\left(f_{j}\right)(\tau-t, \omega, t)\right| .
\end{aligned}
$$

The finite speed of propagation of the wave guarantees that without loss of generality we can assume that

$$
\operatorname{supp}_{y} f(s, y) \subseteq\{|y| \leq 2 t\}
$$

for $0 \leq s \leq t$. Now the application of Lemma 3.1 yields

$$
\left|\mathscr{R}\left(f_{j}\right)(\tau-t, \omega, t)\right| \leq C(1+t)^{3 / 2} \sum_{|\beta| \leq 2}\left\|\Gamma^{\beta} f(t)\right\|,
$$

and we obtain the desired inequality.

This completes the proof. 


\section{APPLication to A SYSTEM OF WAVE AND KLEIN-GoRdon EQUATIONS}

Consider the Cauchy problem

$$
\begin{aligned}
& \square u_{1}=F_{1}(u, \nabla u), \quad(\square+1) u_{2}=F_{2}(u, \nabla u), \quad \nabla=\left(\partial_{t}, \nabla_{x}\right), \\
& u(0, x)=\varepsilon f(x), \quad \partial_{t} u(0, x)=\varepsilon g(x), \quad x \in \mathbf{R}^{3}, \quad \varepsilon>0,
\end{aligned}
$$

where $u=\left(u_{1}, u_{2}\right)$, and analogously for $f$ and $g$. A similar system was considered in [2,3], $f$ and $g$ assumed compactly supported. In contrast to this, here we assume $f, g \in \mathscr{S}\left(\mathbf{R}^{3} ; \mathbf{R}^{2}\right)$. The nonlinearities $F_{j}(u, w)$ are supposed to be smooth in $u, w$; and they have the form $F_{j}(u, \nabla u)=Q_{j}(\nabla u)+$ $C_{j}(u, \nabla u), j=1,2$, where the derivatives of $C_{j}(u, w)$ up to order 2 are zero for $(u, w)=(0,0)$ while

$$
Q_{j}(\nabla u)=\sum_{r, s=0}^{3} \sum_{m, k=1}^{2} q_{j}^{m k r s} \partial_{r} u_{m} \partial_{s} u_{k}
$$

satisfy the strong null condition used in [2], that is

$$
\sum_{r, s=0}^{3} q_{j}^{m k r s} \xi_{r} \xi_{s}=0 \quad \text { for any } \xi=\left(\xi_{0}, \xi_{1}, \xi_{2}, \xi_{3}\right) \in \mathbf{R}^{4}, \quad m, k, j=1,2 .
$$

The above condition simply means that

$$
q_{j}^{m k r s}=q_{j}^{m k s r} .
$$

Examples of quadratic nonlinearities satisfying the strong null condition are discussed in [2]. In order to prove the global existence of a solution of (5.1) for $\varepsilon$ small enough, we introduce the following norms

$$
\begin{gathered}
E_{m}(u(t))=\sum_{|\alpha| \leq m|\beta| \leq 1} \sum_{0 \leq \tau \leq t} \sup _{0 \leq \partial^{\beta} \Gamma^{\alpha} u(\tau) \|,} \\
H_{n}(u(t))=\sum_{|\alpha| \leq n} \sup _{0 \leq \tau \leq t}\left\|(1+\tau+|y|) \Gamma^{\alpha} u(\tau, y)\right\|_{L^{\infty}\left(\mathbf{R}_{y}^{3}\right)} .
\end{gathered}
$$

Denote for the sake of brevity $F_{j}(t, x) \equiv F_{j}(u(t, x), \nabla u(t, x))$ and similarly for $Q_{j}$ and $C_{j}$. To estimate the $L^{2}$ norm of $u_{1}$ we apply the conformal estimate for the linear wave equation due to $S$. Klainerman [11] (see also Chapter VII in [8]),

$$
\left\|\Gamma^{\alpha} u_{1}(t)\right\| \leq C \varepsilon+C \int_{0}^{t} \sum_{|\beta| \leq m-1}\left\|\Gamma^{\beta} F_{1}(\tau)\right\|_{1} d \tau,
$$

where $|\alpha| \leq m$. Further, the energy estimate for the wave equation gives

$$
\left\|\nabla \Gamma^{\alpha} u_{1}(t)\right\| \leq C \varepsilon+C \int_{0}^{t} \sum_{|\beta| \leq m}\left\|\Gamma^{\beta} F_{1}(\tau)\right\| d \tau .
$$


Uniting the above two estimates we get

$$
E_{m}\left(u_{1}(t)\right) \leq C \varepsilon+C \sum_{|\beta| \leq m-1} \sum_{|\gamma| \leq 1} \int_{0}^{t}\left\|\partial^{\gamma} \Gamma^{\beta} F_{1}(\tau)\right\|_{1} d \tau,
$$

since

$$
\Gamma_{j}=\sum_{r, s=0}^{3} c_{j}^{r s} x_{r} \partial_{s}, c_{j}^{r s} \text {-constants, } \quad x_{0}=t .
$$

Similarly, from the energy estimate for the Klein-Gordon equation it follows that

$$
E_{m}\left(u_{2}(t)\right) \leq C \varepsilon+C \sum_{|\beta| \leq m-1} \sum_{|\gamma| \leq 1} \int_{0}^{t}\left\|\partial^{\gamma} \Gamma^{\beta} F_{2}(\tau)\right\|_{1} d \tau .
$$

Since $F_{j}=Q_{j}+C_{j}$, we need estimates of

$$
\left\|\partial^{\gamma} \Gamma^{\beta} Q_{j}(\tau)\right\|_{1} \text { and }\left\|\partial^{\gamma} \Gamma^{\beta} C_{j}(\tau)\right\|_{1}
$$

for $|\beta| \leq m-1,|\gamma| \leq 1$. From the definition of the norms $E_{m}$ and $H_{m}$ it follows that

$$
\left\|\partial^{\gamma} \Gamma^{\beta} C_{j}(\tau)\right\|_{1} \leq C(1+\tau)^{-1} E_{m}(u(\tau)) H_{n}(u(\tau))
$$

provided $n=[m / 2]$ (the greatest integer in $m / 2$ ) and $H_{n}(u(\tau)$ ) is bounded. To estimate the quadratic nonlinearity, we need the following.

Lemma 5.1. If the bilinear form

$$
Q_{j}(\nabla u, \nabla w)=\sum_{r, s=0}^{3} \sum_{m, k=1}^{2} q_{j}^{m k r s} \partial_{r} u_{m} \partial_{s} w_{k}
$$

satisfies the strong null condition, then we have

$$
\left|Q_{j}(\nabla u, \nabla w)(t, x)\right| \leq C(1+t+|x|)^{-1}|\Gamma u(t, x)||\Gamma w(t, x)|,
$$

where $|\Gamma u(t, x)|=\sum_{|\alpha| \leq 1}\left|\Gamma^{\alpha} u(t, x)\right|$.

Proof. If $|x| \leq t+1$, then the desired inequality is obtained in Lemma 2.1 in [2]. Suppose $|x| \geq t+1$. Without loss of generality we can assume $x=\left(x_{1}, 0,0\right)$. From

$$
\Omega_{1 k}=x_{1} \partial_{k}-x_{k} \partial_{1}, \quad k=1,2,3,
$$

we express $\partial_{k}$

$$
\partial_{k}=\left(x_{k} / x_{1}\right) \partial_{1}+\left(1 / x_{1}\right) \Omega_{1 k} .
$$

Analogously, $\Omega_{01}=t \partial_{1}+x_{1} \partial_{t}$ implies $\partial_{t}=\left(-t / x_{1}\right) \partial_{1}+\left(1 / x_{1}\right) \Omega_{01}$. Then setting $\xi_{0}=-t / x_{1}, \xi_{k}=x_{k} / x_{1}, k=1,2,3$, we have the inequality

$$
\begin{aligned}
& \left|Q_{j}(\nabla u, \nabla w)(t, x)-\sum_{r, s=0}^{3} \sum_{m, k=1}^{2} q_{j}^{m k r s} \xi_{r} \xi_{s} \partial_{1} u_{m} \partial_{1} w_{k}\right| \\
& \leq C|\Gamma u(t, x)||\Gamma w(t, x)| /\left|x_{1}\right| .
\end{aligned}
$$


The strong null condition guarantees that the left-hand side of this inequality coincides with $\left|Q_{j}(\nabla u, \nabla w)(t, x)\right|$. Keeping in mind that $\left|x_{1}\right|=|x| \geq t+1$, we complete the proof.

By virtue of this lemma we obtain

$$
\left\|\partial^{\gamma} \Gamma^{\beta} Q_{j}(\tau)\right\|_{1} \leq C(1+\tau)^{-1} E_{m}(u(\tau)) H_{n}(u(\tau)) .
$$

From (5.3) and (5.4) we arrive at

$$
E_{m}(u(t)) \leq C \varepsilon+C \int_{0}^{t} E_{m}(u(\tau)) H_{n}(u(\tau))(1+\tau)^{-1} d \tau .
$$

In order to evaluate $H_{n}\left(u_{1}(t)\right)$ we use the estimate (2.2) for the linear wave equation. Thus, for any $\delta>0$ we have

$$
H_{n}\left(u_{1}(t)\right) \leq C \varepsilon+C \sum_{|\beta| \leq n+3} \sup _{0 \leq \tau \leq t}\left\|\Gamma^{\beta} F_{2}(\tau)\right\|_{3 / 2+\delta} .
$$

In the same way, from the estimate for the linear Klein-Gordon equation due to $T$. Sideris [12] we conclude that

$$
H_{n}\left(u_{2}(t)\right) \leq C \varepsilon+C \sum_{|\beta| \leq n+2} \sup _{0 \leq \tau \leq t}\left\|\Gamma^{\beta} F_{2}(\tau)\right\|_{3 / 2+\delta} .
$$

The quantity $\left\|\Gamma^{\beta} F(\tau)\right\|_{3 / 2+\delta}$ can be estimated by means of Lemma 5.1; this gives the inequality

$$
\left\|\Gamma^{\beta} F(\tau)\right\|_{3 / 2+\delta} \leq C(1+\tau+|x|)^{-1 / 2+\delta} E_{n+3}(u(\tau)) H_{n}(u(\tau)),
$$

provided $E_{n+3}(u(t))$ is bounded. If $n+3=[m / 2]+3 \leq m$, i.e. $m \geq 5$, then we derive

$$
\left\|\Gamma^{\beta} F(\tau)\right\|_{3 / 2+\delta} \leq C(1+\tau+|x|)^{-1 / 2+\delta} E_{m}(u(\tau)) H_{n}(u(\tau)) ;
$$

so

$$
H_{n}(u(t)) \leq C \varepsilon+C \sup _{0 \leq \tau \leq t}(1+\tau+|x|)^{-1 / 2+\delta} E_{m}(u(\tau)) H_{n}(u(\tau)) .
$$

From this estimate and (5.5) following the argument given by S. Klainerman [11] (see also $\S 5$ in [2]), one obtains a global bound for $H_{n}(u(t))+(1+t)^{-\delta} E_{m}(u(t))$. This fact guarantees the existence of a global solution of the system (5.1) assuming $\varepsilon$ small enough.

Another application of our main estimate obtained in Theorem 1 is connected with the Maxwell-Dirac system. This gives us an idea of how to extend the approach of [4] for the case when all the initial data of this nonlinear system are not compactly supported.

\section{REFERENCES}

1. A. Bachelot, Problème de Cauchy global pour des systèmes de Dirac-Klein-Gordon, Ann. Inst. Henri Poincaré 48 (1988), 387-422.

2. V. Georgiev, Global solutions to the system of wave and Klein-Gordon equations, Math. Z. 203 (1990), 683-698. 
3. __ L'existence des solutions globales pour des systèmes nonlinéaires avec champs massifs et sans masse, C. R. Acad. Sci. Paris Sér. I Math. 308 (1989), 529-532.

4. __ Existence des solutions globales pour le système de Maxwell-Dirac, C. R. Acad. Sci. Paris Sér. I Math. 310 (1990), 569-572.

5. S. Helgason, Groups and geometric analysis, Academic Press, New York, 1984.

6. L. Hörmander, On Sobolev spaces associated with some Lie algebras, preprint.

7. $\ldots$, On global existence of solutions of non-linear hyperbolic equations in $\mathbf{R}^{1+3}$, Inst. Mittag-Leffler report no. 9, 1985.

8. __ Non-linear hyperbolic differential equations, Lectures 1986-1987, vol. 2, Lund, 1988.

9. S. Klainerman, Uniform decay estimates and the Lorentz invariance of the classical wave equation, Comm. Pure Appl. Math. 38 (1985), 321-332.

10. _ Global existence of small amplitude solutions to nonlinear Klein-Gordon equations in four space dimensions, Comm. Pure Appl. Math. 38 (1985), 631-641.

11. The null condition and global existence to nonlinear wave equation, Lectures in Appl. Math., vol. 23, Amer. Math. Soc., Providence, RI, 1986, 293-326.

12. T. Sideris, Decay estimates for the three-dimensional inhomogeneous Klein-Gordon equation and applications, Comm. Partial Differential Equations 14 (1989), 1421-1455.

13. W. von Wahl, $L^{\rho}$ decay rates for homogeneous wave equations, Math. Z. 120 (1971), 93106.

Section of Mathematical Physics, Institute of Mathematics, Bulgarian Academy of SCIENCES, 1090 Sofia, Bulgaria 